\title{
Urinary tuberculosis
}

\author{
P. R. RIDDLE \\ Central Middlesex Hospital, Park Royal, London, N.W.10
}

\begin{abstract}
Summary
The present incidence, clinical features and classification of urinary tuberculosis are discussed.

Chemotherapy is the mainstay of treatment. The indications for surgical intervention are reviewed and procedures briefly described.
\end{abstract}

IN A short article it is not possible to go into the detailed minutiae of this subject and only the broad outlines of the present position can be covered.

Although the incidence of tuberculosis as a whole has declined in this country that of urinary tuberculosis stays remarkably steady. The disease is being seen in an older category of patients, and at first presentation the cases are often more advanced. The reason for this is open to conjecture, but since the disease is blood-borne from the lungs, effective treatment of the primary lung lesion may mask or indeed 'cure' the early renal lesions which may then lie dormant for many years before making themselves apparent.

Another feature of the disease is the dramatic decline in the mortality with the introduction of the antituberculous drugs. One series shows a drop in the overall mortality from $57 \%$ in the period 1940 45 , to $2 \%$ in $1960-65$ (Borthwick, 1970). Collected figures in the pre-drug era showed an $80 \%$ 5-year mortality in cases with bilateral renal disease, which dropped to $8 \%$ in the post-drug era (Lattimer, 1955).

With the drop in mortality brought about by drugs there is a steady decline in the need for surgery. Operative intervention is called for, broadly speaking in two categories. In one, during the active phase of the disease, where for instance, progressive narrowing of the lower end of a ureter may so embarrass the kidney, some form of relief may be necessary. A greater need for surgery comes in the second category which includes the late sequelae of the disease. These late manifestations amount really to stricturing at various levels throughout the urinary tract, including the bladder. Urinary tuberculosis in this instance calls for reconstructive surgery.

\section{Classification of the disease process}

There have been various classifications, which are $\overrightarrow{\vec{\omega}}$ mainly useful in prognosis. The disease process is so variable that many categories emerge and classi- $\underset{Z}{7}$ fication then becomes unwieldy. The earliest classi- -3 fication was that of Braasch \& Olson (1919), who relied on urinary tract calcification. They divided of the disease into three categories according to the amount of calcification. Renal calcification varies widely from $7 \%$ (Grenshaw, 1930) to $24.4 \%$ (Gow, $\infty$ 1965). Patients with bladder involvement have beew shown to be more prone to develop calcification and complications (Gow, 1965). Renal calcification musq not be regarded as a static condition, as the calcifie areas can enlarge. These lesions should be followe carefully and excised if necessary. Tuberculous rena $\mathbb{D}$ calcification and stone formation in the same kidne $\vec{\varphi}$ is incidental and rare (Murphy \& Fishbein, 1961

Other classifications have incorporated the bac terial, clinical and radiological findings; such a classification is that of Jacob \& Borthwick (1950). A classification will not be used in this paper, rather the progressive clinical picture will be discussed.

\section{The disease process}

The earliest lesion that can be detected is an asymptomatic positive tuberculous urine culture with no radiological changes in the urinary tract. As it progresses radiological changes in the renal parenchyma, such as cavitation, or narrowing of the minor or major collecting systems, or a combination of both of these, will become evident. Renal parenchymal changes may be difficult or impossible to see in the early stages. Changes in the collecting system are essentially those of stricturing and kinking. This stricturing may be temporary, due to oedema or $\frac{7}{0}$ possibly spasm, or of a more permanent nature with fibrosis and contracture. An example of the former $N$ is shown in Fig. 1.

With the permanent narrowing, progressive 0 fibrosis and contracture of one part of the calyceal system can kink and obstruct the unaffected portion and cause this to dilate. These strictured calyces from읃 time to time become shut off, leaving a closed and 

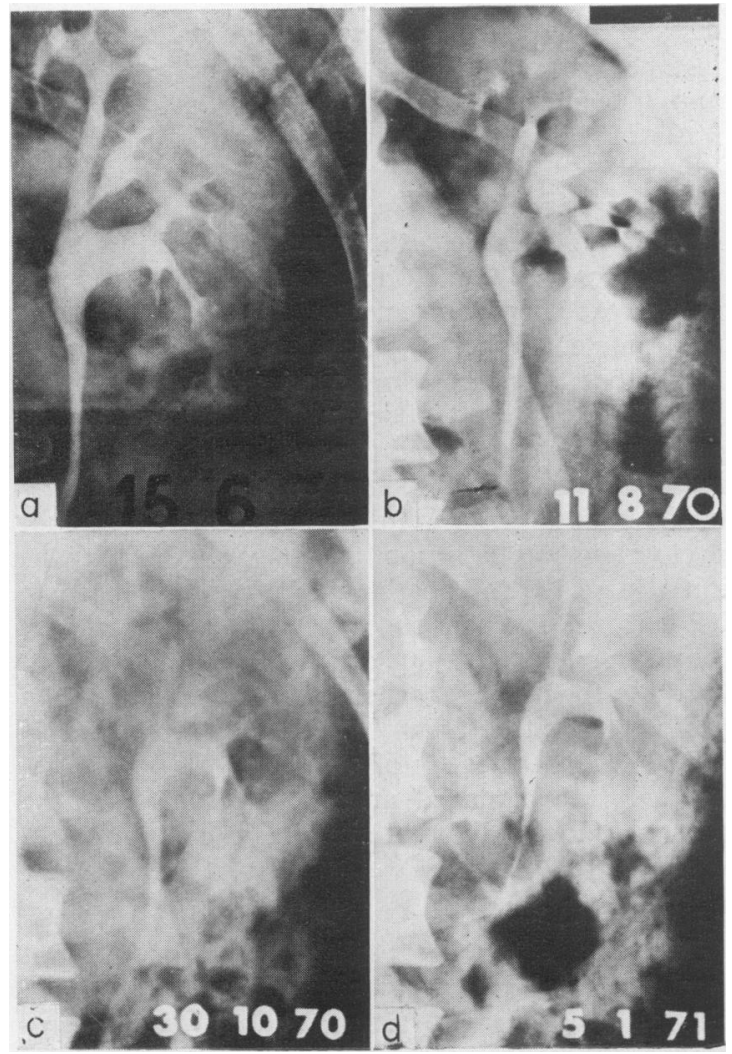

Fig. 1. Temporary narrowing of calyceal neck. (a) 15.6.70. Pyelographic appearances at presentation showing narrowing of middle calyceal neck. (b) 11.8.70. Pyelogram 2 months later showing considerable dilatation of the middle calyx. (c) 30.10.70 Similar though less marked dilatation. (d) 5.1.71. After 6 months of treatment the calyceal dilatation has disappeared.

infected cavity which often increases in size, so destroying surrounding renal parenchyma. It is essential before operating on this latter category to establish that it is completely closed or else a urinary fistula may develop. With progressive pyelous stricturing and kinking the whole kidney may destroy itself without much change necessarily occurring in the rest of the urinary tract. On the other hand, it may be associated with other lesions in the ureter, bladder, or the opposite kidney.

The ureter is commonly invalved, though rarely, if ever, as the sole lesion. With extensive renal involvement virtually the whole ureter may be diseased. The upper and lower ends are the main sites for stricturing, the latter being commoner. It is however, unusual with this isolated lower end 'hold-up' to find active disease in the ureter. Oedema and spasm account for the large number that regress with treatment. Those progressing to severe fibrous stricture must be operated on.
It can be seen that renal parenchymal destruction may occur from the back pressure effect of progressive renal calyceal, pelvi-ureteric or ureteric stricturing. It must not be forgotten, however, that unless the disease causes symptoms, this may happen silently and at the initial presentation the kidney may be functionless.

Bladder involvement usually occurs with extensive upper tract disease, but occasionally may be associated with very minor changes. In early cases the bladder capacity may not be impaired, but with progression it becomes severely reduced. This may not be permanent and on treatment the bladder will very often return to normal (Fig. 2). Unfortunately, fibrosis and contracture of the bladder in a number of patients lead to permanent shrinkage and can leave a small fibrosed organ causing extreme frequency and virtually incontinence. Such cases are usually associated with severe upper tract disease and invariably there are back pressure changes; these are often associated with reflux and secondary infection.

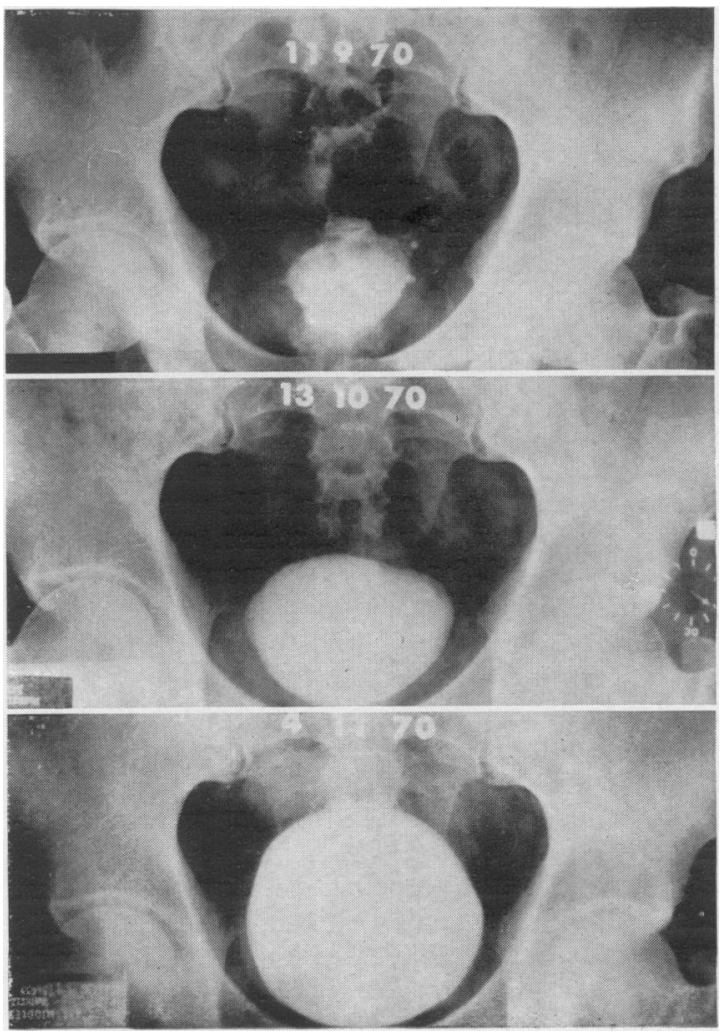

FIG. 2. Temporary bladder changes. Bladder shadow appearances with intravenous pyelography at monthly intervals, showing a progressive improvement with a return to an asympyomatic state. 


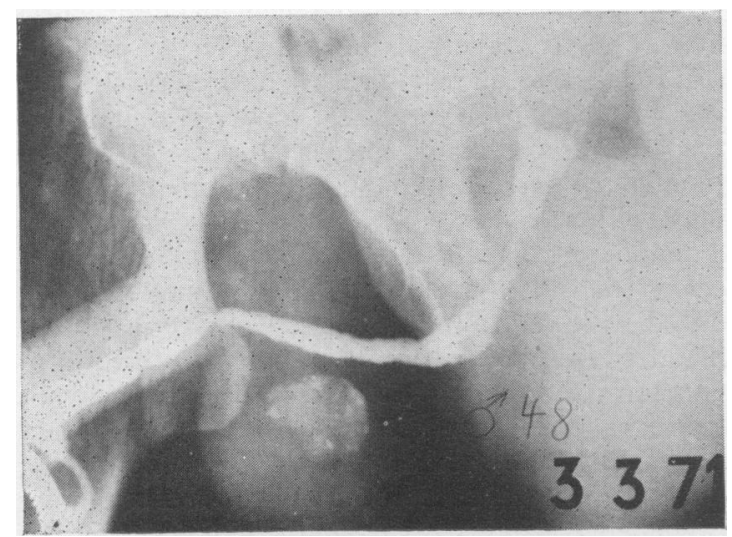

Fig. 3. Urethrogram showing multiple tuberculous urethral strictures.

Urethral involvement is rare and can lead to stricture formation and even fistulae (Fig. 3).

Genital involvement is common and often a calcified vesicle or prostate is seen. Epididymal involvement may lead to sinus formation

\section{Treatment}

In all cases from the mildest to the most severe once the diagnosis has been firmly established, antituberculous chemotherapy is instituted. It is not the purpose of this communication to describe in detail the drug regimes. The mainstay of treatment is the triple drug therapy with streptomycin, PAS (para aminosalicylic acid) and INAH (isonicotinic acid hydrazide). Recently a considerable number of patients have developed drug reactions and resistant strains of bacteria are appearing. For this reason newer drugs, for example rifampicin and ethambutol may have to be employed. However the drug regime is managed, it must continue, once satisfactorily established, for at least 18 months to 2 years. All patients should be admitted, preferably to a sanatorium, during the initial stages of drug stabilization. Mild cases can then be managed at home. Moderate to severe cases should remain at the sanatorium until the disease is well controlled. This is usually the first 3-6 months of the patient's treatment, the period during which surgery may be necessary.

The urine usually becomes negative for acid-fast bacilli during this time. This should be carefully monitored after treatment has finished at increasing intervals but for many years: probably in the region of 10 years.

\section{Surgical treatment}

As mentioned before, surgery falls into two main groups. The first is during the active phase of the disease. This is often extirpatory in nature, as for instance the removal of a totally involved kidney, $\frac{2}{7}$ and indeed the symptoms of frequency may not $\$$ settle until such a kidney is removed. Also in this category is progressive narrowing of the lower end of the ureter which may need temporary relief by means of retrograde catheterization or an intubated 0 ureterostomy in situ. If the bladder is not involved it $\frac{}{0}$ may be expedient to re-implant the ureter early on in $\frac{\bar{S}}{\vec{D}}$ the treatment. Where the bladder is heavily involved $\mathbb{\Phi}$ an intubated ureterostomy can be employed so deferring the question of re-implantation for several months if necessary, in order to allow the bladder to recover. It is thus important to follow the progress $\vec{\omega}$ of the disease very carefully by repeated single-shot o $^{2}$ pyelograms at 1-3-monthly intervals during the initial stages of the treatment. Serial renograms can 3 . be performed when such facilities are available. Not infrequently a kidney can deteriorate and even $i$ become functionless when the interval between $\mathcal{W}$ pyelograms is too long.

The second category of surgery is that of recon- $\vec{\infty}$ structing the damaged urinary tract. This will be 음 discussed at the different sites in the urinary tract $\rightarrow$

\section{Surgery of the kidney}

Reconstructive surgery of the kidney is often ne feasible. Total or partial ablation or drainage of closed-off cavities are the procedures mainly enष्य $\vec{v}$ ployed. Where the kidney is totally or almost totaly. destroyed it is best to remove it. The ureter can be removed down to the bladder so that a blind and often diseased ureteric stump is not left behind. A certain number certainly will reflux into these blind stumps if left and so can cause recurrent urinary infections. With successful anti-tuberculous chemotherapy the removal of the ureter may well be unnecessary (Bloom, Wechster \& Lattimer, 1970). Where a kidney is functionless and particularly when it is completely calcified, there is some controversy as to whether it is safer to leave well alone, rather than to remove it (Bloom et al., 1970; Hanley, 1957). Where one or more calyces have been destroyed by being permanently cut off leaving a closed cavity that often increases in size it is best to relieve the pressure on the adjacent parenchyma by performing the operation of cavernotomy (Hanley, 1957). In this procedure the closed-off pyocalyx is first aspir- $\frac{D}{0}$ ated in order to delineate the roof from the surrounding parenchyma; it is then totally deroofed, $\stackrel{N}{\sigma}$ haemostasis being secured with sutures. Caverno- $N$ tomy can be performed on several cavities in the same $N$ kidney. Where however the fibrosed and closed-off $\sigma$ calyceal neck is contracting so causing infundibular kinking it is necessary to remove this fibrous structure and here a formal partial nephrectomy will be $\stackrel{\mathcal{Q}}{\rightarrow}$ necessary. 
Pelvi-ureteric stricturing is often associated with a useless kidney; in which case a nephrectomy or a nephro-ureterectomy will be necessary. If the kidney is salvagable the usual operations for hydronephrosis are not so successful and a Davis intubation procedure is the operation commonly employed.

\section{Surgery of the ureter}

Isolated narrowings at the lower end are the common strictures to develop in the ureter. They may develop early and rapidly; in which case they are due to oedema or spasm or a combination of both. Such strictures will usually spontaneously recover on treatment. Providing the dilatation is not too great, a wait of several months may be necessary for the oedema and spasm to go. If the dilatation increases rapidly, early intervention will be necessary. The passage of ureteric catheters or stone extractors may shift debris which has collected and enhanced the obstruction at the lower end. Intermittent ureteric dilatation may tide over the time when the obstruction is progressive, though temporary. If this is not successful then a temporary ureterostomy in situ or a direct reimplantation will be necessary. The use of steroids to relieve the oedema at the lower end has been advocated by some; fairly high doses need to be used and if not effective after 2 to 3 weeks should be discontinued.

For permanent strictures some form of reimplantation is necessary. Operations are designed to take into account the length of the stricture and the state of the remainder of the ureter and bladder. Reimplantation is either of the direct variety with or without some form of reflux prevention, or a Boari flap or psoas hitch procedure. It is unusual to need to reimplant into the bladder using a length of ileum or again to need to resort to a transuretero-ureterostomy. It must be remembered that reflux is preferable to stenosis (Fig. 4).

\section{Surgery of the bladder}

Where the bladder is permanently contracted the resultant frequency and even incontinence may be so great that operative intervention is necessary. This can either be reconstructive using some form of cystoplasty, or by means of a urinary diversion. Small contracted bladders are often associated with hold-up at the lower ends of the ureters and vesicoureteric reflux (Fig. 5). It is not unusual for bladder disease of this severity to be associated with marked upper tract lesions; indeed one side has often been totally destroyed or ablated. It is sometimes remarkable how static the appearances are over many years in such cases.

Of the various techniques of cystoplasty available uretero-ileo-caeco-cystoplasty is probably the most attractive because the lower ends of the ureters which are often diseased arealso excised. It alsoincorporates the ileo-caecal valve and this may prevent reflux. Reflux is not a tremendous problem in cystoplasties as the pressures encountered are relatively low.

There are various aspects of the technique, which-

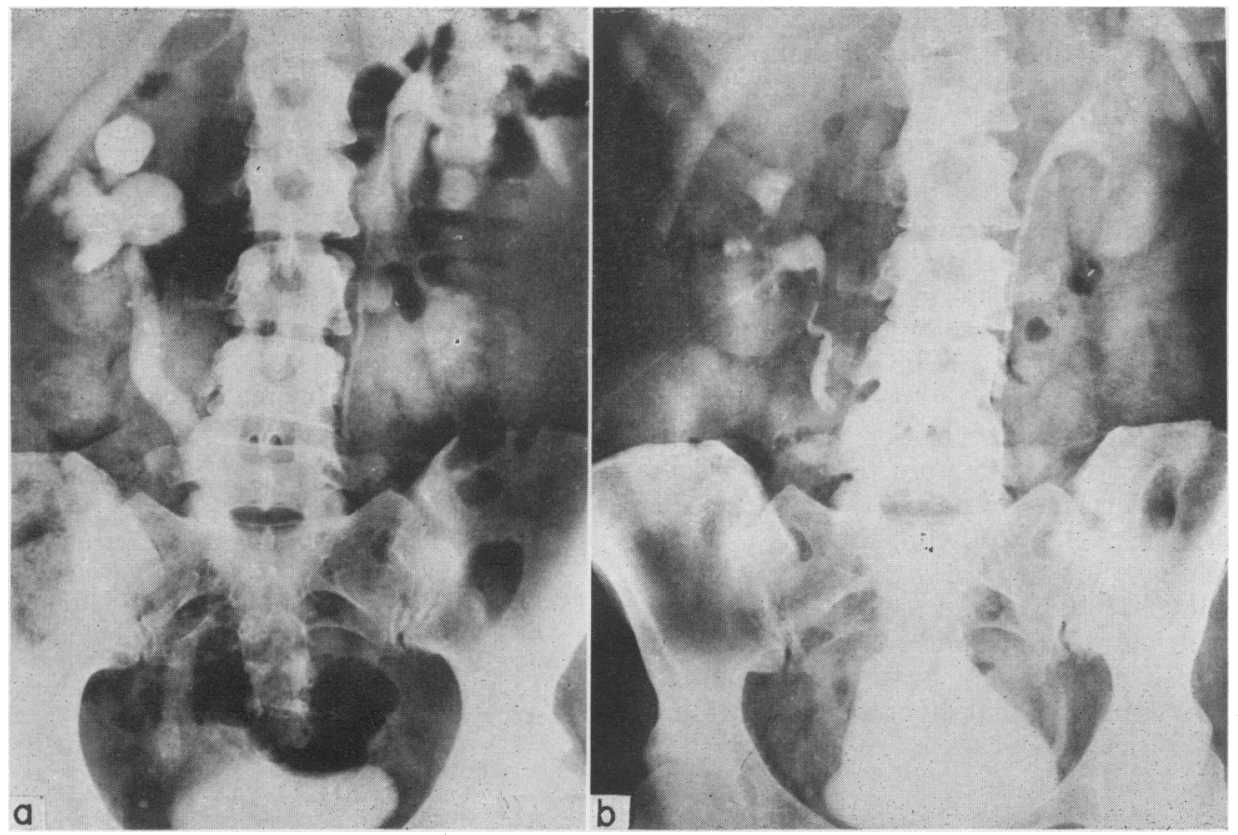

FIG. 4. (a) Intravenous pyelogram showing lower ureteric stenosis unchanged after 9 months of treatment.

(b) Pyelographic appearances 3 months after reimplantation into the bladder. 


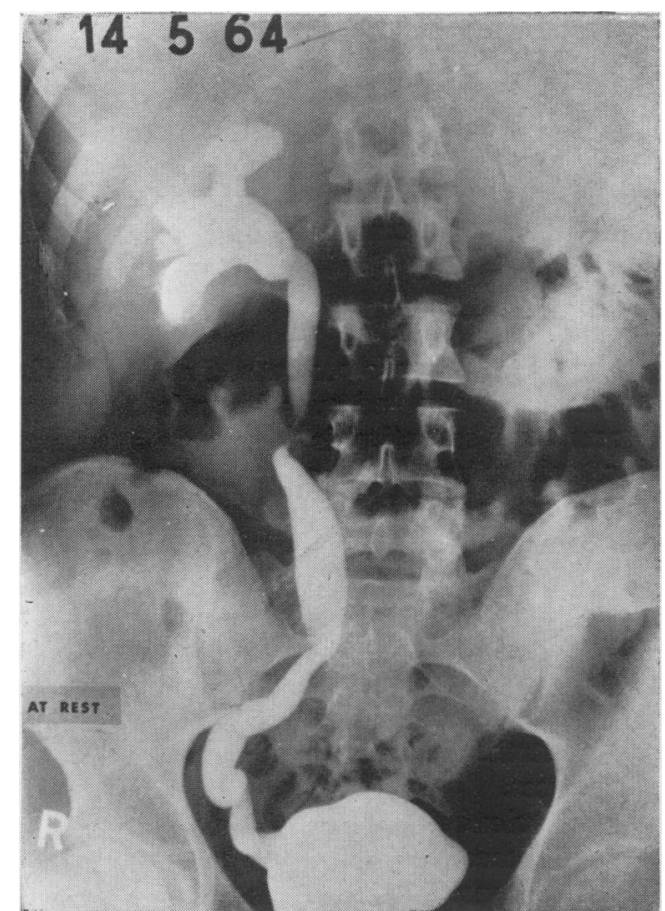

Fig. 5. Cystogram showing contracted bladder and gross reflux up the remaining ureter.

ever organ is used, that are worth mentioning: The bladder must be freely mobilized, care being taken to avoid the lower ends of the ureters if these are to be left in situ. The bladder is excised close to the trigone, leaving the ureters and internal meatus. Free drainage postoperatively is essential both suprapubically and urethrally. Since the voiding pressure is lower the outflow resistance may be relatively high and therefore need reducing. Adjustment of the outflow mechanism will sometimes become necessary (Turner-Warwick \& Handley-Ashkin, 1967).

Urinary diversion may well become necessary. Temporary diversions have already been mentioned. Permanent diversions are to the skin, either directly with a permanent ureterostomy or via a conduit. Bowel diversions are not often practised now-a-days with this condition and are probably unwise as the upper tract is so often already impaired.

\section{Surgery of the urethra}

Urethral stricture is rare and may be associated with fistulae. Strictures may be severe enough to necessitate intermittent dilatations or conventional urethroplasties. In the past urinary diversion has from time to time been employed in this situation. It is probable with modern drug regimes and im- $\frac{3}{\Phi}$

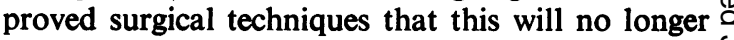
be necessary.

\section{Genital tuberculosis}

Although this paper deals mainly with urinary tuberculosis, urinary and genital tuberculosis are intimately associated. A tuberculous epididymitis may be the first manifestation of the dual disease. Calcification will often be seen in the prostate or $\omega$ vesicles. Tuberculous epididymitis often leads to $\vec{O}$ sinus formation, these rarely heal spontaneously $\vec{\longrightarrow}$ and usually both the testis and the epididymis have $\omega$ to be removed along with the involved scrotal skin.

\section{Conclusions}

Urinary tuberculosis remains at a fairly constant level in the United Kingdom. The disease is best $f$ treated with bed rest and chemotherapy; ablative $y$ surgery is kept to a minimum.

Emergency intervention with upper tract drainage either temporary or permanent may be necessang during treatment.

Careful radiological or renographic monitoring obligatory.

The role of reconstructive urinary surgery is pre minent in the management of the permaneret sequelae of the disease.

\section{References}

BloOM, S., WechSTeR, H. \& LATtimer, J.K. (1970) Results of a long-term study of non-functioning tuberculous kidneys. Journal of Urology, 104, 654.

BoRTHWICK, W.M. (1970) Present position of urinary tuberculosis. British Journal of Urology, 42, 642.

BraAsCH, W.F. \& Olson, E.A. (1919) Roentgenographic diagnosis in renal tuberculosis. Surgery, Gynaecology and $\mathrm{O}$ Obstetrics, 28, 555.

Gow, J.G. (1965) Renal calcification in genito-urinary tuberculosis. British Journal of Surgery, 52, 283.

GRENSHAW, J.L. (1930) Renal tuberculosis with calcification. Journal of Urology, 23, 515.

HANLEY, H.G. (1957) Indications for surgery in renal tuberculosis. British Journal of Surgery, 45, 10.

JACOBS, A. \& BORTHWICK, W.M. (1950) Streptomycin in urinary tuberculosis. Proceedings of the Royal Society of Medicine, 43, 453.

LATTIMER, J.K. (1955) Treatment of tuberculous infections of $\frac{0}{3}$ the genito-urinary tract. Journal of Urology, 74, 291.

MURPHY, G.P. \& FishBeIN, R.H. (1961) Urolithiasis of renal 을 tuberculosis. Journal of Urology, 85, 879.

TURNER-WARWICK, R.T. \& HANDLEY-AsHKEN, M. (1967) The functional results of partial, subtotal and total $N$ caecocystoplasty. British Journal of Urology, 39, 3. 